\title{
Le problème de la preuve en recherche sociologique qualitative
}

Jacques Coenen-Huther

\section{OpenEdition}

Édition électronique

URL : http://journals.openedition.org/ress/380

DOI : 10.4000/ress.380

ISSN : 1663-4446

Éditeur

Librairie Droz

Édition imprimée

Date de publication : 10 décembre 2003

Pagination : 63-74

ISBN : 2-600-00913-2

ISSN : 0048-8046

Référence électronique

Jacques Coenen-Huther, «Le problème de la preuve en recherche sociologique qualitative », Revue européenne des sciences sociales [En ligne], XLI-128 | 2003, mis en ligne le 11 novembre 2009, consulté le 19 avril 2019. URL : http://journals.openedition.org/ress/380 ; DOI : 10.4000/ress.380 
Jacques COENEN-HUTHER

\title{
LE PROBLÈME DE LA PREUVE EN RECHERCHE SOCIOLOGIQUE QUALITATIVE
}

\author{
SOMMAIRE: 1. Explication ou diagnostic sociologique? - 2. Logique de \\ découverte et logique de vérification. - 3. La capacité prédictive comme élément \\ de preuve. -4 . Complémentarité possible des approches. -5 . Une logique auto- \\ nome. -6 . L'ambiguïté de l'échange verbal. -7 . Représentativité ou typicalité. - \\ 8. Pour conclure.
}

Les chercheurs en sociologie se répartissent bon gré mal gré en «quantitativistes» et «qualitativistes». Certains d'entre eux récusent pourtant cette distinction, arguant que la recherche empirique ne peut se passer de quantification mais que ce qui se quantifie n'épuise pas le réel. S'il ne s'agissait que d'un choix purement technique, on pourrait en effet en contester l'opportunité. Confronté aux rapports que les humains établissent entre eux, l'esprit de découverte néglige le nombre et la fréquence. Comme l'écrivait William Foote Whyte, "In the first stage of exploration, everything seems new and strange, but eventually I see a pattern that seems to make sense. Once I have described that pattern in writing, I lose interest in seeing the pattern repeated again and again and look for a new field to explore" (Whyte, 1994, pp. 4-5.) Mais ultérieurement, la nécessité d'apprécier la fréquence ainsi que le caractère majoritaire ou minoritaire d'un «pattern» de comportement finit par s'imposer. Le recours au chiffre est dès lors inévitable. Il pourrait donc paraître souhaitable que les chercheurs de terrain maîtrisent toute la gamme des techniques de recherche. La priorité accordée aux techniques quantitatives ou qualitatives découlerait alors de choix ad hoc.

Mais l'importance accordée à la quantification dans la recherche sociologique ne peut se ramener purement et simplement à des considérations techniques bien que celles-ci ne puissent être négligées. Le sort fait à la quantification résulte le plus souvent de conceptions épistémologiques et méthodologiques différentes ainsi que d'attitudes différentes face au «fardeau de la preuve» et à la nécessité d'établir des garde-fous de méthode visant à apporter une connaissance vérifiée. Si l'on garde à l'esprit l'opposition sommaire entre les disciplines qui disposent de procédures de preuve standardisées et celles qui ne peuvent se fonder que sur le recoupement des témoignages et l'argumentation des interprétations, on ne peut que conclure que la communauté scientifique des sociologues ne cesse d'être écartelée entre ces deux pôles. Certes, en matière d'établissement de la preuve, aucun sociologue ne peut prétendre rivaliser avec les mathématiciens ou les physiciens. A cet égard, le caractère strictement scientifique de la sociologie relève davantage de l'idéal proclamé et de l'intention tenace que d'une réalité 
établie sans conteste ${ }^{1}$. Très nombreux cependant sont les sociologues qui répugnent à l'admettre et qui continuent à se référer au modèle des sciences de la nature ou à une caricature de ce modèle. Ils se sentent rassurés par la standardisation des techniques et la quantification des résultats. Le recours au chiffre leur permet d'éluder la réalité inconfortable de la relation circulaire du sociologue à son objet. L'usage des techniques d'échantillonnage, le calcul des marges d'erreur, la construction de plans expérimentaux ou quasi-expérimentaux, l'emploi de techniques d'analyse multivariée, la mise en œuvre de tests statistiques: tout cela donne le sentiment de se mouvoir sur un terrain solide et de progresser vers toujours plus de scientificité.

Pourtant, tout comme leurs collègues «qualitativistes», les sociologues «quantitativistes » sont confrontés à un problème général de validité, même s'il est partiellement dissimulé par une confusion très fréquente entre validité des techniques et validité des résultats ${ }^{2}$. Tout comme leurs collègues qualitativistes, ils se trouvent face à «un objet qui parle», qui est sujet à des attentes de «désirabilité» et dont la crédibilité est loin d'être toujours assurée. Tout comme leurs collègues qualitativistes, ils doivent se défendre d'un biais subjectif pouvant se manifester dans le découpage des catégories d'analyse ou dans les si fréquentes explications ex post. Et pour autant qu'ils soient lucides à propos de leurs propres travaux, ils doivent bien se rendre à l'évidence que le «modèle du laboratoire » la reproduction à l'identique de résultats par des chercheurs différents sous des conditions constantes - est, dans la plupart des cas, tout à fait inadéquat dans la mesure ou sa mise en œuvre ne peut être tenue pour un objectif réaliste.

En dépit de ces difficultés qui sont au cœur de la recherche sociologique quelles qu'en soient les modalités - et qui tiennent à la nature même de l'entreprise sociologique - les chercheurs privilégiant les techniques qualitatives se retrouvent régulièrement en position défensive dans les débats entre sociologues. Nonobstant les facteurs d'incertitude qui affectent leurs travaux, les quantitativistes se voient comme ceux qui «prouvent» ce qu'ils affirment: les chiffres sont là! Ils font généralement preuve de scepticisme face aux comptes rendus de recherches qualitatives car ils n'y trouvent pas les instruments de preuve auxquels ils sont accoutumés. Il en résulte une attitude générale de condescendance à l'égard de leurs collègues qui ne s'expriment que sur le mode narratif. Il est vrai que la recherche qualitative est fréquemment le refuge de chercheurs peu soucieux de méthode, paraissant croire qu'il suffit de «goûter le spectacle de la vie » et de se fier à des intuitions non contrôlées pour faire œuvre authentiquement sociologique ${ }^{3}$. Mais plutôt que de rendre sarcasme pour sarcasme et d'invoquer «l'esprit de finesse» des uns s'opposant à «l'esprit de géométrie» des autres, essayons ici de voir de quelle manière se comporte la recherche sociologique qualitative face à l'exigence de tenir un discours rationnel sur la réalité sociale et d'apporter une connaissance partiellement détachable de son contexte d'origine.

Je me permets de renvoyer sur ce point à mon article récent, La sociologie des sciences entre positivisme et sociologisme (2002, p. 221.)

Voir à ce sujet mon livre A l'écoute des humbles (2001, pp. 25 et ss.)

Je reprends ici l'argumentation développée dans mon Observation participante et théorie sociologique (1995, pp.173 et ss.) 


\section{EXPLICATION OU DIAGNOSTIC SOCIOLOGIQUE?}

Observons tout d'abord que le souci exagéré de la preuve peut avoir quelque chose de dérisoire ou de paralysant. Une part notable de la recherche sociologique consiste, non pas à établir des rapports de causalité, mais à poser des diagnostics, c'est-à-dire à décrire dans un langage spécifique des réalités perçues par tout un chacun ${ }^{4}$. Quand un médecin baptise «extrasystole» la contraction cardiaque éprouvée par son patient, il n'explique rien, il ne propose aucune relation de cause à effet; il se borne à traduire les propos du patient dans un langage spécialisé. L'exercice n'est pourtant pas futile pour autant. Il suppose une connaissance préalable du fonctionnement du muscle cardiaque et il oriente la pensée vers un certain type d'explication. Il en va de même du diagnostic sociologique qui applique à certains faits un mode de conceptualisation favorisant la généralisation - c'est-àdire l'élargissement contextuel - et privilégiant certaines variables structurelles. Cet exercice de traduction n'est pas justiciable d'une procédure de preuve mais il est porteur d'un élargissement cognitif, tant pour le sociologue lui-même que pour le profane. Donnons-en ici un exemple. Lorsqu'une observation participante de longue durée m'a amené à présenter le kibboutz en tant que système social, comme un ensemble bifonctionnel, à la fois communauté de vie et communauté de travail (1995, p. 24), je n'ai fait qu'émettre par là un truisme ne nécessitant aucune vérification Mais cette vérité d'évidence m'a conduit à faire usage des fameuses pattern-variables parsoniennes, à savoir les dichotomies universaliste/particulariste, spécifique/diffus, affectif/affectivement neutre, fondé sur la performance/ascriptif (ibid.). L'application de cette taxinomie préétablie permettait de conclure par voie logico-déductive - mais congruente avec les résultats de l'observation - à l'existence de deux systèmes de rôles aux exigences partiellement contradictoires et dont la juxtaposition ne pouvait qu'être une source de tensions perceptibles dans différents domaines de la vie du kibboutz: un système de rôles régi par les normes universalistes qui sont de mise entre camarades de travail et un sytème de rôles régi par les normes particularistes qu'il est normal de trouver entre parents ou quasi-parents (ibid., pp. 24-25.) Une première décontextualisation consista à présenter les tensions observables comme contenues en germe dans le projet socio-politique initial combinant une communauté de vie et une communauté de travail tout en liant l'ensemble à un mode de gestion démocratique. Cette contradiction interne au projet initial rendait compte de la crise généralisée qui affectait la société kibboutznique. Une deuxième décontextualisation consista à s'affranchir du cadre initial pour proposer l'idée que toute organisation fondée sur des systèmes de rôles difficilement compatibles ne peut qu'être sujette à des crises. On a donc ici une argumentation émergeant non de la manipulation de données mais bien de la mise en œuvre de procédés de conceptualisation. Son domaine de validité s'élargit à mesure qu'on s'écarte de l'objet initial pris dans sa singularité et qu'on le redéfinit de manière plus abstraite en se fondant

Je me réfère à ce propos à la distinction établie entre «diagnostic sociologique » et «explication sociologique» proposée par Hans Zetterberg. Cet auteur note que "To make a sociological diagnosis of the subject-matter or problem $X$ is to describe $X$ in terms of a sociological taxonomy." (1965, pp. 24-29.) 
sur une caractéristique formelle comme la bifonctionnalité. Ce raisonnement offre une perspective spécifique qui écarte des interprétations singularisantes et des jugements moralisateurs en mettant l'accent sur l'existence de conditions structurelles qui pèsent sur la volonté des acteurs. A aucun moment ne sont impliquées des hypothèses qu'il conviendrait de vérifier ou de réfuter. Néanmoins, l'argumentation débouche indirectement sur une structure causale: alors que les kibboutzim étaient en crise - ceci n'avait pas à être prouvé par le chercheur - un facteur explicatif de cet état de crise était identifié.

\section{LOGIQUE DE DÉCOUVERTE ET LOGIQUE DE VÉRIFICATION}

Lorsque la stratégie de recherche est celle de l'analyse comparative continue (constant comparative analysis), pratiquée par Glaser et Strauss (1965, 1973), la preuve de ce qu'on avance est fournie graduellement par la cohérence générale de la théorie qui émerge progressivement des observations de terrain - ce qu'on appelle la grounded theory - et par le mode de conceptualisation adopté, très proche des perceptions des acteurs concernés. Ces auteurs estiment en effet que la contribution du sociologue est optimale lorsqu'il présente ses observations d'une manière qui «sonne juste» pour les personnes familiarisées avec le terrain, mais aussi d'une manière qui n'aurait pas été adoptée par ces personnes ${ }^{5}$. Dans cette proximité assortie d'un décalage apparaît l'apport spécifique du sociologue. Celui-ci offre en effet une connaissance contextualisée mais partiellement détachable de son contexte d'origine. C'est dans leur ouvrage Awareness of Dying (1965), compte rendu d'une observation dans un hôpital où sont traités des patients en phase terminale, qu'ils présentent avec le plus de clarté leur stratégie de recherche. Leur unité d'observation dans cette recherche est la «situation d'interaction ». Entre patients et personnel de santé s'observent des situations d'interaction qui sont aussi des «contextes de conscience»(awareness contexts). Le patient peut ne pas se rendre compte qu'il va bientôt mourir alors que tout le monde autour de lui en est conscient; c'est la closed awareness ou conscience fermée $^{6}$. Il peut arriver que le patient soupçonne ce que les autres savent, sans toutefois en être certain; on se trouve dans le suspected awareness context ou contexte de conscience présumée. Il arrive que le patient se définisse comme mourant, que le personnel de santé ait la même perception, mais que chacun fasse semblant de ne rien savoir: il s'agit alors de la mutual pretense awareness ou conscience feinte mutuelle. Il y a aussi le cas où le patient et le personnel sont conscients de la fin prochaine et interagissent en conséquence; c'est l'open awareness ou conscience ouverte $(1965$, p. 11.) La solidité de la théorie émergente est fournie par la saturation de l'observation: pour autant qu'on s'en tienne à une opposition dichotomique entre le patient et le personnel de santé pris globa-

Ce qu'ils expriment de la manière suivante: “...in such a fashion that his account rings true to insiders, but also in such a fashion that they themselves would not have written it ..." (1965, pp. 8-9.)

6 On adopte ici les traductions proposées par Henri Peretz dans l'ouvrage publié par Isabelle Baszanger (1992, p. 114. 
lement ${ }^{7}$, on est confronté à quatre - et seulement quatre - contextes de conscience. Le tout forme un ensemble logique dont les transformations sont soumises à des règles bien précises: le contexte de conscience feinte mutuelle, par exemple, ne peut évoluer que vers le contexte de conscience ouverte (ibid., p. 74.) De l'évolution des différents contextes de conscience résultent également des «styles de mort» (styles of dying) distincts qui deviennent très rapidement prévisibles ${ }^{8}$. L'émergence graduelle d'un corps de connaissance fiable en l'absence de tout instrument de preuve propre au «modèle du laboratoire » résulte précisément de la mise en œuvre d'une logique de découverte distincte de la logique de vérification animant la recherche quantitative ${ }^{9}$. La logique de vérification classique se déroule selon des plans expérimentaux ou quasi-expérimentaux et procède par vérification d'hypothèses portant sur les variables impliquées. La logique de découverte progresse au contraire selon un modèle arborescent où chaque fait d'observation supplémentaire affine et précise l'état antérieur de la théorie en voie d'élaboration. Le processus comporte donc un élément d'auto-correction qui se substitue aux procédés de vérification reposant sur la loi des grands nombres.

\section{LA CAPACITÉ PRÉDICTIVE COMME ÉLÉMENT DE PREUVE}

Il existe donc des séquences de travail de terrain dont les résultats trouvent leur vérification dans le mouvement même de la recherche. A cet égard, l'analyse d'une situation d'interaction relativement simple, assortie des commentaires d'informateurs $^{10}$, peut acquérir une valeur prédictive qui vérifie a posteriori le diagnostic posé. Ainsi, me trouvant à Moscou au cours de la dernière année de l'URSS chancelante, je fus amené à la suite d'un événement fortuit à m'intéresser au déroulement des files d'attente si intimement liées au système de distribution de type soviétique. Je découvris rapidement, contrairement à ce que mon univers de référence occidental me donnait à penser, qu'il s'agissait bel et bien d'un collectif émergent, producteur d'une auto-organisation récurrente jouant le rôle de substitut fonctionnel à une organisation efficace de la distribution des biens et services $^{11}$. Mes informateurs russes m'initièrent aux normes de comportement régissant ces files d'attentes et qui en faisaient bien davantage que le simple système d'interdépendance de fait auquel j'étais habitué et qui n'implique ni rôles ni attentes de rôles spécifiques ${ }^{12}$. Chacun se devait de bien visualiser la personne qui le précédait et la personne qui le suivait. Ceci permettait au moins en principe

Et à l'exclusion des interventions éventuelles du milieu familial.

8 Une stratégie de recherche comparable à celle de Glaser et Strauss a été mise en œuvre en France par Demazière et Dubar (1997.)

9 Cette distinction a été présentée de manière convaincante par Glaser et Strauss (1973, pp. 12-15.)

10 Au sens ethnologique du terme.

11 J'ai développé tout ceci en détail dans un article paru initialement dans la Revue française de sociologie (1992) et repris dans Observation participante et théorie sociologique (1995, chap. VI.). Voir aussi à ce sujet le commentaire de Jean-Michel Berthelot (1996, pp. 229-231.)

12 Je fais allusion ici à la distinction entre systèmes d'interdépendance et systèmes fonctionnels opérée par Raymond Boudon (1979.) 
d'éviter toute contestation concernant l'ordre de la queue. Une fois le rang établi par confirmation de la personne qui précédait, de la personne qui suivait, ou des deux à la fois, on était libre de s'absenter un moment pour vaquer à d'autres occupations. Pour obtenir la reconnaissance de son droit, on s'adressait de préférence à la personne qui suivait: celle dont le rang aurait été menacé par l'insertion dans la file d'un élément non autorisé. Les répliques indicatives d'attentes de rôles étaient brèves, elliptiques et stéréotypées. Elles pouvaient être enseignées au débutant et témoignaient d'une routinisation accentuée de ces pratiques (1995, pp. 146-148.) Dès le moment où j'eus noté et mémorisé ces échanges verbaux typiques, il me devint possible non seulement de prévoir ce qui se allait se passer dans des contextes similaires mais aussi d'interagir de manière efficace et «socialement compétente» dans des situations d'interaction du même genre. On a donc ainsi une forme de preuve par la participation même aux rapports sociaux qu'on se donne pour tâche d'étudier et l'on peut faire l'économie de toute procédure formelle de vérification. La réduction significative de l'élément de «double contingence» propre à toute situation d'interaction est suffisante pour établir la validité de la connaissance acquise.

\section{COMPLÉMENTARITÉ POSSIBLE DES APPROCHES}

Ceci dit, les chercheurs qualitativistes ne peuvent échapper à l'obligation d'assumer le «fardeau de la preuve» que s'ils acceptent d'être cantonnés dans un rôle subalterne par rapport à la recherche dite quantitative. C'est le cas - très fréquent - lorsqu'une séquence qualitative est conçue comme la phase exploratoire d'un processus de recherche plus diversifié culminant dans une collecte de données effectuée sur le mode quantitatif. On peut se satisfaire d'un tel statut auxiliaire et même renverser l'ordre de préséance. C'est au fond ce que suggère implicitement la déclaration de Whyte citée plus haut: le chercheur de pointe explore des domaines peu familiers, met à jour un complexe comportemental nouveau, s'efforce d'en comprendre le sens, puis va de l'avant et laisse à d'autres le soin de faire de la «science normale» et d'apprécier l'importance et la fréquence des phénomènes observés. Il m'est arrivé d'adopter cette attitude lorsque j'ai analysé les modalités d'ouverture ou de fermeture du domicile ${ }^{13}$. J'ai notamment suggéré que l'ouverture fréquente du domicile peut revêtir des significations très différentes et correspondre à des motivations diverses, parfois radicalement divergentes. Dans le cas du domicile «salon», baptisé ainsi en reconnaissance d'une inspiration proustienne, le domicile est utilisé comme instrument de sociabilité de manière plus consciente qu'ailleurs. On invite fréquemment et l'on se plaît en une compagnie nombreuse. Dans l'ordre des valeurs, primauté est accordée ici à la sphère publique. Les personnes qui invitent ont des ambitions dans la sphère publique et recherchent l'accroissement de leur force sociale par le truchement des invitations. Il n'y a pas dans ce cas de recherche de symétrie dans les prestations. Au contraire, tout le jeu des invitations réside dans la création de relations

13 Renvoyons sur ce point à un article paru dans les Cahiers internationaux de sociologie (1991) et repris également dans mon Observation participante ... (1995, chap. IV.) 
au moins temporairement asymétriques (1995, pp. 105-106.) Le domicile «foyer» est également conçu comme un lieu de sociabilité. Mais contrairement au cas précédent, ce sont les normes de la sphère privée qui donnent le ton. On organise moins de réceptions ou de repas à couverts nombreux et davantage de rencontres de couple à couple. L'intention première est de découvrir dans de nouveaux rôles des personnes pour lesquelles on nourrit de l'intérêt. Il s'agit d'une stratégie d'élargissement du contexte initial d'une relation, orientée vers le loisir et opérant en référence première à la sphère privée. Le domicile garde sa fonction première de foyer; cette fonction s'élargit au cercle des amis que l'on encourage à se sentir «comme chez eux». Les préoccupations de prestige et de pouvoir sont absentes. En revanche, à l'inverse du cas précédent, les relations s'organisent sur des attentes de symétrie (ibid., pp. 107-109.) Les différents rapports au logement mis en évidence par la recherche dans six cas de figure différents - le domicile «salon», le domicile «foyer», le domicile «forum», le domicile «moulin», le domicile «champ clos» et le domicile «refuge» correspondent à des logiques sociales bien établies. Ces logiques, séparément et globalement, font sens et sont aussi accessibles à l'observateur que les contextes de conscience dégagés par Glaser et Strauss; les procédures formelles de vérification n'ont pas davantage leur raison d'être ici. En revanche, les relations esquissées entre les rapports au logement et le statut social reposent sur une base empirique réduite. Elles gagneraient à être mises à l'épreuve d'une vérification extensive grâce à des techniques standardisées.

\section{UNE LOGIQUE AUTONOME}

Loin d'être toujours dans un rapport de complémentarité vis-à-vis de l'analyse quantitative, le chercheur qualitativiste peut préserver l'autonomie de sa démarche lorsqu'il s'agit de dégager le sens de certaines pratiques et de mettre en évidence des complexes d'interaction. La fréquence des phénomènes observés est alors dénuée de pertinence. Ainsi en est-il dans le cas des travaux de Glaser et Strauss qui se passent de tout recours au chiffre. Dans une telle démarche, la quantification ne peut en aucun cas renforcer la validité des résultats et Jean-Claude Kaufmann a raison de mettre en garde contre les tentatives dérisoires d'élaborer des pseudomesures sur une base empirique inadéquate (1996, p. 30.) La recherche sociologique qualitative a une vocation particulière à s'appuyer sur des données hétérogènes et c'est la cohérence d'ensemble offerte par ces données hétérogènes - résultats d'observation, données d'entretien, conversations entendues et notées, indications de témoins privilégiés, documents d'archives, extraits d'œuvres littéraires - qui constitue la meilleure garantie de sérieux ${ }^{14}$. L'établissement de la preuve s'apparente alors à ce qu'il peut être en histoire. Ceci est d'autant plus compréhensible que les questions qu'on se pose en sociologie ne peuvent souvent trouver réponse adéquate que sur fond de connaissance historique. C'est ce qui a poussé à plusieurs reprises Giovanni Busino à plaider pour un rapprochement de la

${ }_{14}$ Je m'appuie ici sur l'introduction de mon livre A l'écoute des humbles (2001, pp. 26-27) 
sociologie et de l'histoire ${ }^{15}$ et ce qui a conduit Jean-Claude Passeron a parler de l'histoire et de la sociologie comme de deux disciplines «discernables sociologiquement» bien qu'étant «épistémologiquement indiscernables» (1990, p. 12.) Un sociologue cherchant à comprendre l'état actuel des rapports sociaux dans une haute vallée alpine ne pourra qu'y voir le résultat d'un processus de changement social rapide. En gros, deux événements majeurs ont contribué à la physionomie sociale actuelle de ces régions: au $19^{\mathrm{e}}$ siècle, l'arrivée des alpinistes anglais et la demande de guides de haute montagne qu'elle a suscitée; au $20^{\mathrm{e}}$ siècle, la construction des grands barrages qui a créé l'accès à de nouveaux emplois salariés tout en améliorant les communications avec la plaine. Les rapports de force actuels entre les groupes de parenté - en termes de patrimoine, de contrôle des ressources et d'influence politique - s'expliquent au moins en partie par la manière dont les individus et les groupes ont réagi à ces deux événements et s'y sont adaptés. Les raisonnements pouvant être présentés en la matière s'appuient à la fois sur des informations recueillies auprès des habitants et sur l'observation de leurs comportements mais aussi sur des données à caractère historique. Tout ceci forme un ensemble diversifié; c'est la convergence des éléments d'appréciation qui constitue le critère de vérité le plus convaincant. Comme en recherche historique, toute information particulière est confrontée à des données accessoires tenant lieu de circumstantial evidence et ce sont les indices de plausibilité ainsi recueillis qui se substituent aux procédures formelles de vérification. Comme certaines données se vérifient dans le cours même de la recherche, les informations fournies par les habitants sont rendues crédibles - et se confirment les unes les autres - par la participation même à la vie de la région lorsque le sociologue s'y immerge suffisamment. Il faut bien voir en effet que les opérations de recherche qui se révèlent comme telles ne font que court-circuiter le jeu normal des interactions sociales. Comme un informateur le fit observer à Whyte, "if people accept you, you can just hang around, and you'll learn the answers in the long run without even having to ask the questions" (1994, p. 75.) Ceci revient à dire que le facteur temps est un élément dans l'établissement de la preuve et que le choix de la «temporalité d'investigation » est partie intégrante de la stratégie de recherche, comme le soulignent à juste titre Arborio et Fournier (1999, pp. 30-31.) Le déroulement normal de la vie sociale n'apporte ses révélations que dans le long terme. Par rapport à ce rythme lent, la recherche sociologique accélère le mouvement. Mais il convient de noter à ce propos une différence appréciable entre la recherche quantitative et la recherche qualitative. Cette dernière s'inscrit malgré tout dans la durée et ne peut arriver à des résultats probants que si elle est sous faible contrainte de temps. En comparaison, la recherche quantitative constitue l'accélérateur par excellence, avec toutes les limites que cela implique.

\section{L'AMBIGUÏTÉ DE L'ÉCHANGE VERBAL}

Les sociologues font actuellement un grand usage de la parole des acteurs, au point qu'on a pu ironiser sur la «sociologie du magnétophone» (Cuin, 2000,

15 Voir notamment son ouvrage intitulé La permanence du passé (1986). 
p. 144) qui se borne à reproduire de façon hâtive les propos des acteurs. Hors, comme tout échange verbal, l'entretien de recherche comporte une part irréductible d'ambiguïté qui ne cesse de susciter des interrogations, des doutes, et qui appelle fréquemment une exigence de preuve. Ayant à traiter des données d'entretien, le chercheur ne peut échapper à la nécessité de préciser le statut qu'il entend leur conférer. La parole est-elle, elle-même, le fait à prendre en considération? La parole renvoie-t-elle à un autre fait qui reste à définir ou à circonscrire? La parole dissimule-t-elle au contraire un fait qui se dérobe à l'analyse? Dès lors, les propos recueillis seront-ils considérés comme des informations pertinentes en soi ou seront-ils tenus au contraire pour les révélateurs de configurations mentales ou comportementales sous-jacentes? (Coenen-Huther, 2001, pp. 9-10) La réponse à ces questions qu'on ne peut éluder est fortement dépendante de l'objectif visé par la recherche. Par voie de conséquence, le type de preuve à attendre en dépend également. Un même entretien peut fournir en effet des données d'ordre divers. Il peut apporter des informations concrètes sur des manières d'agir; il peut offrir des éléments d'interprétation d'une certaine configuration sociale ou socio-économique; il peut enfin refléter un certain état d'esprit (ibid., pp. 25-26.) Cette diversité des apports et des usages de l'entretien est fréquemment à l'origine de malentendus quant au mode de preuve attendu. Dans la plupart des cas, les propos tenus n'ont qu'une valeur stratégique indirecte. Quelle que soit leur exactitude factuelle, ils sont les révélateurs d'un état d'esprit, d'une mentalité, d'une attitude face à la vie ou, plus directement, d'une appartenance sociale ou culturelle. L'exactitude des faits évoqués aura alors moins d'importance que le caractère typique ou atypique de la mentalité qui s'est exprimée dans la narration. Un des personnages mis en scène dans mon livre A l'écoute des humbles (2001, chap. 2) est un ancien berger des hauts pâturages du Jura. Je l'ai appelé Guillaume pour les besoins de la publication. Guillaume ne cesse d'insister sur la dureté de la vie d'autrefois. Même s'il se pose en victime du sort, il est assez fier de pouvoir évoquer la vie rude qu'il a menée et de pouvoir décrire des conditions de travail difficiles que les jeunes ont peine à imaginer de nos jours. Ce faisant, il ne résiste pas toujours à la tentation d'exagérer ses performances, qu'il s'agisse de pommes de terre à arracher, de foin à faucher ou de stères de bois à couper. Il livre ainsi des informations qui ne sont pas toujours à prendre au pied de la lettre. Son témoignage comporte incontestablement une part d'exagération, entretenue pour des raisons d'équilibre psychique personnel. Il fut possible de confronter les déclarations de Guillaume avec des informations provenant d'autres sources : des voisins, des amis, un médecin, un travailleur social. Ces recoupements - rendus nécessaires par l'exigence de vérification à laquelle l'enquêteur ne peut se soustraire portèrent sur une série de données factuelles et permirent de mieux comprendre la logique de présentation de soi de l'intéressé. Mais au-delà de cette dimension personnelle, la logique sous-jacente aux propos recueillis reste la donnée essentielle du point de vue de la recherche sociologique. Cette logique s'exprime dans le contexte particulier d'une relation d'interaction avec le chercheur, en fonction d'attentes de rôles et d'attentes de rôles perçus. Cette contextualisation du discours n'a cependant trait qu'à sa forme, non au message qu'il véhicule. 


\section{REPRÉSENTATIVITÉ OU TYPICALITÉ}

La recherche quantitative vise généralement à la représentativité. Par rapport à une population $N$, un sous-groupe $n$, obtenu grâce à un échantillonnage aléatoire ou par quotas, est censé en offrir une image en miniature. La recherche qualitative, au contraire, a pour objectif de sélectionner des individus qui soient typiques d'une certaine catégorie de personnes. Ce caractère typique ne peut être établi que par la prise en compte d'éléments externes au cas étudié. Un autre personnage présenté dans mon livre A l'écoute des humbles, Marco, ouvrier sicilien immigré en Suisse, peut être considéré comme typique d'une catégorie de travailleurs du sud de l'Europe qui se sont présentés sur le marché du travail en Suisse, à une époque où la demande de main d'œuvre était très forte dans certains secteurs d'activité, notamment la construction et la restauration (Coenen-Huther, 1995, chap. 1.) Ce qui permet d'établir la typicalité de Marco, c'est, bien entendu, ce qu'on sait de lui, mais c'est aussi tout ce qu'on a appris antérieurement sur les ouvriers immigrés en général et les immigrés du sud de l'Europe en particulier. Jusqu'où peut-on aller dans l'affirmation du caractère typique de notre homme? Après son arrivée à Genève, Marco se met à fréquenter assidûment bars, discothèques et boîtes de nuit. Ce comportement participe-t-il de sa typicalité ou est-il le fruit d'une idiosyncrasie? La transplantation de Sicile en Suisse est aussi, dans ce cas, le passage d'un milieu villageois à un milieu urbain. Ainsi, Marco se trouve coupé des formes de sociabilité auxquelles il était habitué - les copains avec qui on traîne sur la place du village - et il s'efforce d'en reconstituer l'équivalent dans un autre contexte. C'est ce qui le projette dans la vie nocturne de la grande ville. De cette façon, on se retrouve à nouveau entre copains, mais ces copains sont tous des Italiens. Il y a là une situation d'exclusion et d'auto-exclusion sociale et culturelle fréquente qui se prolonge pendant de nombreuses années. Certains comportements qu'on serait tenté de prime abord de soustraire à la typicalité générale et de tenir pour révélateur d'une certaine personnalité peuvent donc être ramenés à la condition d'immigré. Contrairement à la représentativité statistique qui s'obtient en amont de l'analyse sociologique, le caractère typique ou atypique d'un comportement ne peut être établi qu'à la suite d'un raisonnement sociologique.

Lorsque la collecte de cas typiques s'insère dans une stratégie de recherche fondée sur la comparaison constante continue, la recherche d'individus typiques obéit à la logique arborescente évoquée plus haut. Chaque nouveau cas de figure émergent suscite le besoin de trouver un contre-exemple aux fins de comparaison. Contrairement à l'échantillonnage visant à la représentativité globale, on pratique alors ce qu'il est convenu d'appeler «l'échantillonnage théorique» (theoretical sampling). Ce sont les besoins de la grounded theory émergente qui guident à chaque étape la sélection des individus (Glaser et Strauss, 1973, chap. 3.)

\section{POUR CONCLURE}

En sociologie, la recherche qualitative et la recherche quantitative se rejoignent au moins sur un point: le «modèle du laboratoire» transposé du domaine des sciences de la nature est contre-productif en ce sens qu'il impose au chercheur des exigences impossibles à satisfaire. Bien sûr, à l'exemple de nombreux socio- 
logues, on peut continuer à s'y référer comme à un idéal symbolisant «l'intention de science» de la sociologie. Néanmoins, cette attitude n'est sans risque ni pour le projet scientifique de la sociologie ni pour la santé mentale du chercheur individuel dans la mesure où elle place celui-ci dans une situation de décalage perpétuel - et aisément perceptible - avec les normes d'activité qu'il se donne pour tâche de respecter. Comme l'écrivait Alvin Gouldner, "when sociologists commit themselves compulsively to a life-wasting high science model, they are making a metaphysical wager. They are wagering that the sacrifice is «best for science.» Whether this is really so, they cannot confirm; but they often need no further confirmation than the pain this self-confinement inflicts upon them." $(1970,1972$, p. 506.) Dans l'état actuel des enseignements de méthode, il faut parfois toute une vie pour se débarrasser d'illusions positivistes persistantes et pour se réconcilier avec une conception plus réaliste de la recherche sociologique. Il n'est pas rare que ce déniaisement progressif s'accompagne de démoralisation ou de cynisme. Pourtant, comme le note Passeron, « un raisonnement sociologique ne peut être de part en part ni de bout en bout un raisonnement expérimental.» (1991, p. 78.) Pour qu'il puisse le rester à l'état pur, il faudrait qu'il n'énonce que des trivialités sur le monde qui nous entoure. De tout cela, il faut bien le dire, les praticiens de la recherche qualitative sont généralement plus conscients que leurs collègues quantitativistes. Toutefois, ayant perdu l'assise apparemment solide qu'offrent les données chiffrées et les techniques standardisées, ils sont guettés par un risque inverse: celui d'une nonchalance méthodologique tolérant toutes les dérives de type journalistique. Si le chiffre n'est plus l'ultime recours de la scientificité, tout n'est-il pas permis? Il est bon que les uns et les autres prennent conscience du fait que «l'administration de la preuve ne peut jamais revêtir complètement en sociologie le forme logique de la «falsification» au sens poppérien» (Passeron, 1991, p. 63.) Mais on ne peut que souhaiter que les mirages scientistes ne soient pas remplacés par l'amateurisme. Dans l'ambiance générale de relativisme cognitif qui a gagné les sciences sociales, on peut nourrir des craintes à ce sujet. Discipliner le raisonnement exige autant de rigueur et d'intégrité intellectuelle que dompter le chiffre.

Carouge/Genève

\section{RÉFÉRENCES BIBLIOGRAPHIQUES}

Arborio, Anne-Marie et Fournier, Pierre, L'enquête et ses méthodes: l'observation directe. Paris, Nathan, Coll. "128”, 1999.

Baszanger, Isabelle, Ed., La trame de la négociation. Sociologie qualitative et interactionnisme. Paris, L'Harmattan, Coll. «Logiques sociales », 1992.

Berthelot, Jean-Michel, Les vertus de l'incertitude. Le travail de l'analyse dans les sciences sociales. Paris, Presses universitaires de France, Coll. «Sociologie d'aujourd'hui », 1996.

Boudon, Raymond, La logique du social. Paris, Hachette, 1979.

Busino, Giovanni, La permanence du passé. Genève, Droz, Coll. «Pratiques sociales et théories», 1986.

Coenen-Huther, Jacques, Le domicile: sphère publique et sphère privée, «Cahiers internationaux de sociologie», Vol. XCI, 1991, pp. 301-313. 
Coenen-Huther, Jacques, Production informelle de normes; les files d'attente en Russie soviétique, «Revue française de sociologie», Vol. XXXIII, No 2, 1992, pp. 213-232.

Coenen-Huther, Jacques, Observation participante et théorie sociologique. Paris, L'Harmattan, Coll. «Logiques sociales», 1995.

Coenen-Huther, Jacques, A l'écoute des humbles. Entretiens en milieu populaire. Paris, L'Harmattan, Coll. «Logiques sociales», 2001.

Coenen-Huther, Jacques, La sociologie des sciences entre positivisme et sociologisme, «Revue internationale des sciences sociales », Tome XL, No 124, pp. 219-231, 2002.

Cuin, Charles-Henry, Sociologie sans paroles : Durkheim et le discours des acteurs, in: M. Borlandi et M. Cherkaoui, Eds, Le Suicide. Un siècle après Durkheim. Paris, Presses universitaires de France, 2000.

Demazière, Didier et Dubar, Claude, Analyser les entretiens biographiques. L'exemple de récits d'insertion. Paris, Nathan, Coll. "Essais et Recherches", 1997.

Glaser, Barney G. et Strauss, Anselm L., Awareness of Dying. Chicago, Aldine Publishing Company, 1965.

Glaser, Barney G. et Strauss, Anselm L., The Discovery of Grounded Theory. Chicago, Aldine Publishing Company, 1973.

Gouldner, Alvin W., The Coming Crisis of Western Sociology. Londres, Heinemann, 1970, paperback edition, 1972.

Kaufmann, Jean-Claude, L'entretien compréhensif. Paris, Nathan, Coll. “128”, 1996.

Passeron, Jean-Claude et Prost, Antoine, L'enseignement, lieu de rencontre entre historiens et sociologues, «Sociétés contemporaines », No 1, mars 1990, pp. 7-45.

Passeron, Jean-Claude, Le raisonnement sociologique. L'espace non-poppérien du raisonnement naturel. Paris, Nathan, Coll. "Essais et Recherches", 1991.

Whyte, William Foote, Participant Observer. An Autobiography. Ithaca, New York, IRL Press, 1994.

Zetterberg, Hans L., On Theory and Verification in Sociology. Totowa, N.J., The Bedminster Press, 1965. 